\title{
Multi-hop Hierarchical Routing Based on the Node Health Status in Wireless Sensor Network For Forest Fire Monitoring
}

\author{
A. Anhar \\ Department of Electronic and Computer Engineering \\ Brunel University \\ London, United Kingdom \\ anhar.anhar@brunel.ac.uk
}

\author{
R. Nilavalan \\ Department of Electronic and Computer Engineering \\ Brunel University \\ London, United Kingdom \\ rajagopal.nilavalan@brunel.ac.uk
}

\begin{abstract}
This paper proposed multi-hop hierarchical routing to monitor forest fire using wireless sensor network. The node health status is introduced in choosing Cluster Head (CH) in order to avoid route breakages caused by $\mathrm{CH}$ that burns off. $\mathrm{CHs}$ transmit their data to the closest $\mathrm{CH}$ which is in the direction to the base station. The performance of proposed method is compared to the Leach, MTE, and the direct algorithm. The extensive simulation is done by NS2 with results showing that the proposed method outperforms others in term of the number of packets received, energy consumed, the number of the alive nodes, and average end to end delay.
\end{abstract}

Keywords-multi-hop; hierarchical; node health status; forest fire; Leach;

\section{INTRODUCTION}

Wireless Sensor Network (WSN) which connecting sensor nodes and Base Station have been implemented in many areas ranging from military until environment. These huge number of applications can be classified into two groups: monitoring and tracking [1]. One of the environmental applications of WSN is to monitor forest fire [2]. Compare to other technologies such as satellite-based systems and digital camera; this network has many advantages. It is more cost-effective to install many sensor nodes in wide area monitoring in order to increase its scalability. Furthermore, it has a fast response and is not influenced by weather or vision.

Since sensor nodes are equipped with limited batteries, the energy issue is the main concern of many researchers. In forest fire monitoring, sensor nodes commonly deploy in a rural area, and therefore it is impossible to replace the battery when it is exhausting. In addition, the lower delay should be achieved when there is a fire in the monitored area. In short, the energy consumed, the lower average delay, as well as the throughput, are the critical factors in designing wireless sensor network.

Routing technique is a method to choose paths in sending data from sensor nodes to the destination or base station. Recently, researchers have shown an increased interest in proposing routing algorithms to enhance the performance of
WSN. According to [3], based on the way how the sensor nodes are connected to others, the routing protocol can be grouped into two types: flat protocol and hierarchical protocol. Every sensor node has the same responsibilities in flat protocol whereas in hierarchical, there are some nodes which are selected as $\mathrm{CHs}$ to gather data from their member and transmit these data to the base station.

The routing protocol in WSN is application-specific. Due to every application has the different requirements, the design of routing protocol should consider these demands to a robust system. In the critical application such as forest fire monitoring, the reliable data delivery and low delay are the main consideration in designing routing protocol as well as energy efficiency. Node died because of the sensed attribute should be deemed as a key factor to improve the network reliability in WSN. Furthermore, as the coverage area of this application is wide, the routing protocol should gain high scalability to transmit data to the BS which is far away from sensor nodes.

There are many proposed routing protocol for forest fire monitoring. However, none of those considers the node health status as a parameter to select $\mathrm{CH}$. In this work, the hierarchical protocol which is grouping sensor nodes based on the health status is implemented to monitor forest fire. It can increase robustness in the network as a result of no dead $\mathrm{CH}$ caused by burning process in the forest. Moreover, sensor nodes in dangerous status have higher priority in transmitting packets. Therefore the average end to end delay of the cluster will be low. To improve the scalability, the proposed multihop routing is developed where it combines the hierarchical and minimum transmission energy routing.

The remainder of this paper is organized as follow. Section 2 discusses Leach protocol as a fundamental of hierarchical routing and others which are implemented in a critical environment such as forest fire detection. Section 3 explains the proposed method in this study. Parameters and results of a simulation using Network Simulator 2 are examined in section 4. Finally, section 5 concludes the paper and future works of this research. 


\section{RELATED WORKS}

Although Low Energy Adaptive Clustering Hierarchy (Leach) protocol is not specially designed for forest fire monitoring, it is a fundamental of hierarchical routing which is introduced by W. Heinzelman, et., all [4]. Leach chooses CHs in a distributive way in the set-up phase whereas in the steadystate phase every member of $\mathrm{CH}$ sends their packets to $\mathrm{CH}$. These two phases accomplish a round in Leach. At every beginning of round, sensor node $i$ can elect itself as a $\mathrm{CH}$ with probability $P_{i}(t)$ which is formulated as

$$
P_{i}(t)=\left\{\begin{aligned}
\frac{k}{N-k *\left(r \bmod \frac{N}{l}\right)}, & C_{i}(t)=1 \\
0, & C_{i}(t)=0
\end{aligned}\right.
$$

Where $k$ is the expected number of clusters per round, $N$ is the total number of nodes in the network, and $r$ is the number of rounds which have already passed. CHs send an advertisement to other sensor nodes, and sensor nodes choose the best $\mathrm{CHs}$ which are close to them. Every sensor node transmits its packets to the $\mathrm{CH}$ using TDMA schedule, and $\mathrm{CH}$ forwards the aggregation packets to the base station. Leach assumes that all sensor nodes can directly transmit their data to the Base Station with enough power if needed as consequent LEACH is not suitable to be applied to the network with large coverage area.

Threshold-sensitive Energy Efficient sensor Network (TEEN) and Adaptive Periodic TEEN (APTEEN) are designed for time-critical application such as forest fire which is proposed in [5][6]. TEEN is the hierarchical reactive routing while APTEEN is hierarchical hybrid routing. Both algorithms assume that base station has global information of the network, so it selects $\mathrm{CH}$ according to energy and distance with simulated annealing. TEEN introduce Hard and Soft Threshold referring to the value of the sensed attribute. Sensor node will send data to the $\mathrm{CH}$ if the sensed value is higher than Hard Threshold or differ from the previous sensed value by equal or greater than Soft Threshold. As a result, the TEEN algorithm does not transmit packets continuously. On the other hand, APTEEN implements not only Hard/Soft Threshold but also the query routing. Under those circumstances, APTEEN is a complex routing protocol.

The author in [7] presented Environmental Monitoring Aware (EMA) routing protocol which considers node failure due to the sensed environments and takes into account the health status of the network. EMA is a flat routing and the decision criteria to select the path according to the RSSI, hop count, and the node health status. Although EMA can provide the robustness in delivering data, it is not hierarchical routing. As the number of node increase, there will be many packets in the network and routing table become bigger. Accordingly, the average end to end delay will rise significantly.

Jamil, et., al [8] introduced Maximise Unsafe Path (MUP) routing for disaster situations. The basic idea of MUP is to deliver data to the dangerous area in order to utilize the energy of dangerous node. There are four types of the node health status: safe, low safe, unsafe, and almost-failed. MUP is a flat routing and not suitable for higher density node because of the higher end to end delay.
Y. Ha in [9] proposed the cluster-base routing protocol for fire monitoring. The main idea of this technique is to generate and restructure the sensor node network cluster hierarchically based on the fire propagation over the network cluster to reduce the number of transmission of the fire data from the sensor node to the Base Station. There are two stages of $\mathrm{CH}$ namely: Master $\mathrm{CH}$ and Slave $\mathrm{CH}$. The slave $\mathrm{CHs}$ send their data to the master $\mathrm{CH}$, and the master $\mathrm{CH}$ sends its data and the data from the slave CHs to the Base Station. In one period, there is only one the master $\mathrm{CH}$, and the others are slave $\mathrm{CHs}$. The prerequisites to become a master $\mathrm{CH}$ are:

- A CH which first time is detecting fire.

- The lowest number of transmission to the Base Station.

- The closest distance to the Base Station.

- The least number of sensors detecting fire.

- A CH with the highest remaining energy.

The energy efficiency of EFMP is achieved by minimizing the number of transmission times compares to the traditional cluster-base routing such as LEACH and TEEN by choosing the master head in case of the fire. However, this routing has a high overhead because of the number of packets in the process of electing the master $\mathrm{CH}$.

\section{PROPOSED METHOD}

Before proceeding to describe the proposed method, it is important to explain two conditions in selecting a $\mathrm{CH}(\mathrm{CH})$ as shown in fig. 1 . The normal condition is a situation where there is no fire close to $\mathrm{CH}$ as shown in (a). All CH's members send their data to the $\mathrm{CH}$, and it relays these data to the Base Station (BS) after performing aggregation. This $\mathrm{CH}$ cannot be chosen as a $\mathrm{CH}$ for the next round in order to distribute energy consumed in the network. When at a certain round a selected $\mathrm{CH}$ is a sensor node which is near to the fire, some data from this cluster cannot be transmitted to the BS since this $\mathrm{CH}$ is going to die at an unpredicted time. Furthermore, if this $\mathrm{CH}$ is an intermediate node which forward data from other $\mathrm{CH}$ to base station, there will be many data loss in the network. The proposed method avoids choosing this node as a $\mathrm{CH}$ and guarantees no data losses (which are necessary sensing data because there is a fire around them) in that cluster.

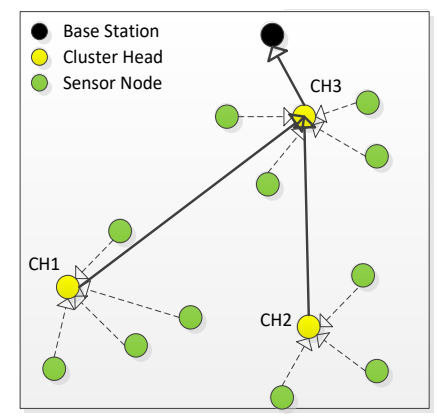

(a)

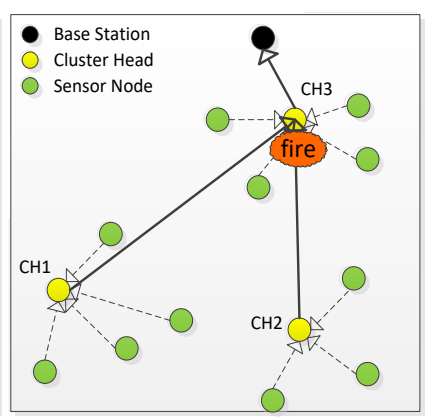

(b)
Fig. 1.The impact of choosing a danger node as a $\mathrm{CH}$

Essentially, the proposed method has the same stages as Leach protocol, which is divided into two stages: set-up stage and steady-state stage. A sensor node is eligible to be selected 
as a $\mathrm{CH}$ if the health status of the node is safe and its probability fulfils the probability in Leach algorithm. Following that, CHs advertise its position to other sensor nodes and $\mathrm{CHs}$. $\mathrm{CHs}$ which receive these ads decide the next hop $\mathrm{CH}$ using Minimum Transmission Energy (MTE) algorithm [4]. At the same time, sensor nodes choose the best $\mathrm{CH}$ according to distance. A sensor node in dangerous status has a high priority to send a joint request message to the $\mathrm{CH}$. A TDMA schedule is sent by $\mathrm{CH}$ and member of cluster transmit their packets to the base station. If the base station is far away from the $\mathrm{CH}$, it forwards the data to the next hop $\mathrm{CH}$ until the data arrive at the base station.

The node health status of a sensor node in the proposed method refers to the condition of sensor node whether it is close to the fire or not. Three states of node health status are:

1. Safe: Sensor node which is far away to the fire

2. Danger: Sensor node which is close to the fire

3. Dead: Dead node because of fire

Energy model used in this scheme has the same pattern as in Leach routing. The amount of energy consumed to transmit $l$ bit of data on distance $\mathrm{d}\left(\mathrm{E}_{\mathrm{Tx}}\right)$ is formulated as:

$$
\begin{aligned}
& E_{T x}=l_{x} E_{\text {elect }}+l \cdot \varepsilon_{f g} \cdot d^{2} \quad \text { for } 0 \leq d \leq d_{\text {crossover }} \\
& E_{T X}=l_{.} E_{\text {elect }}+l_{\text {mp. }} d^{4} \quad \text { for } 0 \geq d_{\text {crossover }}
\end{aligned}
$$

Where $E_{\text {elect }}$ is energy consumed for electronic processing, $\varepsilon_{\mathrm{fs}}$ and $\varepsilon_{\mathrm{mp}}$ are constant for free space propagation when the distance is lower than $\mathrm{d}_{\text {crossover }}$ and constant for multi-path propagation when the distance is higher than $\mathrm{d}_{\text {crossover }}$ respectively. On the other hand, energy spent in the receiver is:

$$
E_{R x}=l_{.} E_{\text {elect }}
$$

\section{SIMULATION SCENARIO AND RESULTS}

To analyze the performance of the proposed method, Network Simulator (NS) 2.35 is used with Leach protocol extension from MIT. NS is open source software used for developing and analyzing a new protocol as stated in [10][11] both for a wired and wireless network. It is an event driven simulator which supporting many protocols in MAC and Network Layer.

The scenario of sensor nodes in danger status is created at some interval certain rounds to analyze the effect of choosing these nodes as CHs. These interval times must be set up before the beginning time of the round. Nodes in danger status will die at randomly interval time. The proposed method is compared with three others method such as Leach, direct, and MTE. The direct method sends sensing data of sensor nodes directly to the Base station while MTE routing is a multi-hop technique choosing the next-hop neighbour based on the closest node that is in the direction to the BS.

In our experimental environment, the numbers of sensor nodes are 100 and placed in the area $100 \times 100 \mathrm{~m}^{2}$ randomly. Base Station (BS) is located at position $(50,175)$ and it assumes that every sensor node starts with the equal energy. TwoRayGround and Mac/Sensor is a type of propagation and
Mac layer which is implemented in this simulation. We modify packet.cc and packet.h file to add the node health status in the header of the packet. In addition, ns-leach.tcl from MIT must be reorganized in order to fit with the proposed approach. The detail of simulation parameters can be found in table 1.

TABLE I. SIMULATION PARAMETERS

\begin{tabular}{|l|c|}
\hline \multicolumn{1}{|c|}{ Parameters } & Value \\
\hline Propagation & TwoRayGround \\
\hline MAC Layer & 100 \\
\hline Number of nodes & 50,175 \\
\hline Location of BS & $100 \times 100$ \\
\hline Topology size & $2 \mathrm{~J} / 4 \mathrm{~J}$ \\
\hline Initial Energy & $1000 \mathrm{~s} / 100 \mathrm{~s}$ \\
\hline Simulation time & \\
\hline
\end{tabular}

There are two scenarios in the simulation. To investigate the energy and number of data received, we simulate for 1000 second with $2 \mathrm{~J}$ initial energy. The first and the second node died at $3^{\text {rd }}$ and $9^{\text {th }}$ round. On the other hand, to calculate the average delay, we set up a simulation for 100 seconds with $4 \mathrm{~J}$ initial energy. Therefore, there will be no dead node caused by energy drain during simulation time. Only one node die because of fire at $3^{\text {rd }}$ round.

Fig. 2 illustrates total data received in BS over the time when the number of the burnt nodes is two. There is a sharp rise in the number of data received for all of the methods until a certain time, and it will remain stable because all nodes died. The comparison result of the proposed method, Leach, MTE, and direct also showed that proposed method had achieved superior performance in term of data received in the sink. The proposed method guarantees that there are no missing packets as result of no dead CHs. The MTE routing has the lowest number of packet received because every packet traverses multi hops to reach the BS.

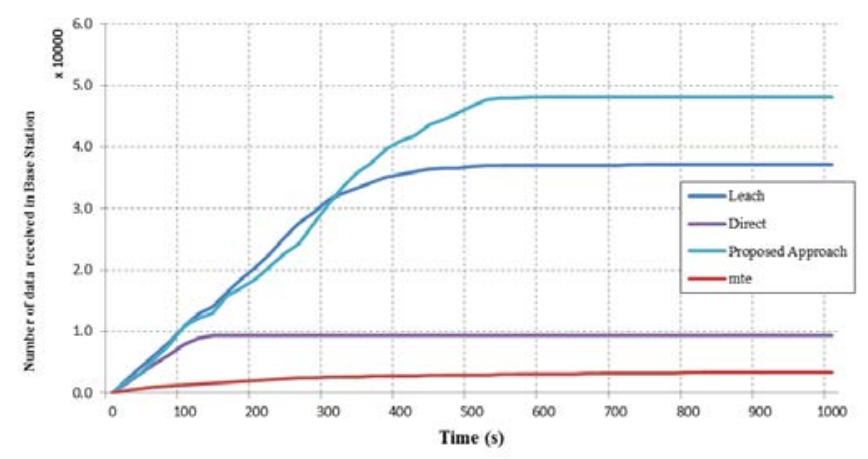

Fig. 2. The number of data received in BS vs time.

Fig. 3 compares the number of nodes alive over the time, and fig. 4 explores the total energy consumed over the time for four methods. Generally speaking, proposed method beats others in these two cases. Leach and proposed method have 


\section{Draft Version - Brunel University}

one node alive at around 660 seconds. On the other hand, MTE has the longest life time since the numbers of data sent are very small. With regards to energy, there is a gradual rise of energy spent in Leach, MTE, and proposed method while it rose sharply in the direct method as shown in fig. 6. All methods spent 200J maximum energy since there are a hundred nodes with 2J initial energy. Leach, MTE, and proposed method utilized 198J at approximately 1000 seconds whereas the direct spent 200J at around 140 seconds. The direct method spent more energy than others because of its distance to the BS and no aggregation process in the sensor nodes.

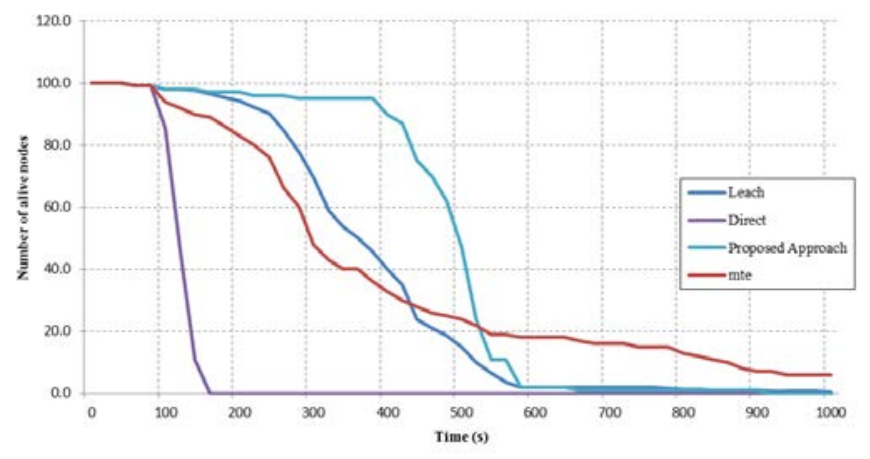

Fig. 3. The number of alive nodes vs time.

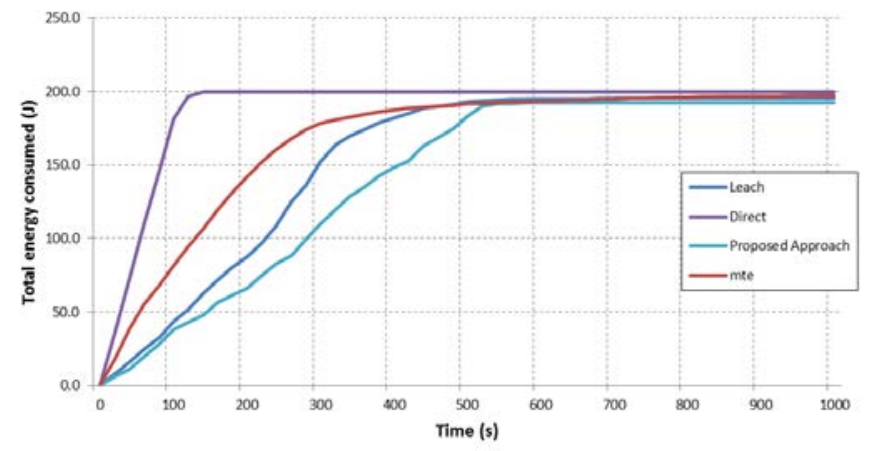

Fig. 4. Total energy consumed vs time.

With regard to the average end to end delay, the proposed approach has the lowest value owing to the priority in the cluster which has a dead node. As shown in table 2, the proposed approach experiences $39.75 \mathrm{~ms}$ while leach, MTE, and direct algorithm are $64.72 \mathrm{~ms}, 94.37 \mathrm{~ms}$, and $205.92 \mathrm{~ms}$ respectively. The high delay end to end in direct method and MTE is resulted from the number of packets travelling in the network without aggregation process such in hierarchical routing.

TABLE II. AVERAGE END TO END DELAY

\begin{tabular}{|c|c|c|c|c|}
\hline & \multicolumn{4}{|c|}{ Protocol } \\
\cline { 2 - 5 } & Proposed Approach & Leach & MTE & Direct \\
\hline $\begin{array}{c}\text { Average Delay } \\
\text { (ms) }\end{array}$ & 39.75 & 64.72 & 94.37 & 205.92 \\
\hline
\end{tabular}

\section{CONCLUSION}

This paper aims to enhance the throughput as well as delay end to end of the wireless sensor network deployed for forest fire monitoring with considering the possibility of node failure because of burnt activities. We have developed the proposed approach in the multi-hop hierarchical network and included the node health status as a variable to select CHs. Future work will focus on the further optimization of clustering of sensor nodes and comparison with other routing in wireless sensor network.

\section{ACKNOWLEDGMENT}

The authors wish to thank the Directorate General of Resources for Science, Technology and Higher Education Ministry of Research, Technology and Higher Education of Indonesia for providing A. Anhar a PhD scholarship to pursue his study at Brunel University London, UK.

\section{REFERENCES}

[1] P. Rawat, K. D. Singh, H. Chaouchi, and J. M. Bonnin, "Wireless sensor networks: A survey on recent developments and potential synergies," $J$. Supercomput., vol. 68, no. 1, pp. 1-48, 2014.

[2] A. a. a. Alkhatib, "A Review on Forest Fire Detection Techniques," Int. J. Distrib. Sens. Networks, vol. 2014, pp. 1-12, 2014.

[3] N. A. Pantazis, S. A. Nikolidakis, and D. D. Vergados, "Energy-Efficient Routing Protocols in Wireless Sensor Networks: A Survey,” IEEE Commun. Surv. TUTORIALS, vol. 15, no. 2, 2013.

[4] W. B. Heinzelman, A. P. Chandrakasan, and H. Balakrishnan, "An application-specific protocol architecture for wireless microsensor networks," IEEE Trans. Wirel. Commun., vol. 1, no. 4, pp. 660-670, 2002.

[5] M. Ghiasabadi, M. Sharifi, N. Osati, S. Beheshti, and M. Sharifnejad, "TEEN: a routing protocol for enhanced efficiency in wireless sensor networks," 2008 Second Int. Conf. Futur. Gener. Commun. Netw., vol. 1, no. C, pp. 2009-2015, 2001.

[6] Manjeshwar A., D. P. Agrawal, and a Manjeshwar, “ APTEEN: A hybrid protocol for efficient routing and comprehensive information retrieval in wireless sensor networks,” Int. Parallel Distrib. Process. Symp., vol. 00, no. C, pp. 195-202, 2002. 
[7] B.-L. Wenning, D. Pesch, A. Timm-Giel, and C. Görg, "Environmental monitoring aware routing: making environmental sensor networks more robust," Telecommun. Syst., vol. 43, no. 1-2, pp. 3-11, 2009.

[8] A. Jamil, D. J. Parish, R. C.-W. Phan, I. Phillips, J. Whitley, and G. Oikonomou, "Maximise unsafe path routing protocol for forest fire monitoring system using Wireless Sensor Networks,” 2012 IEEE 3rd Int. Conf. Networked Embed. Syst. Every Appl., pp. 1-8, 2012.

[9] Y. G. Ha, H. Kim, and Y. C. Byun, “Energy-efficient fire monitoring over cluster-based wireless sensor networks,” Int. J. Distrib. Sens. Networks, vol. 2012, 2012.

[10] W. Kasch, J. Ward, and J. Andrusenko, "Wireless network modeling and simulation tools for designers and developers,” IEEE Commun. Mag., vol. 47, no. 3, pp. 120-127, 2009.

[11] T. Issariyakul and E. Hossain, Introduction to Network Simulator (NS) 2, Second. Springer Science \& Business Media, 2012. 